\title{
Vertical Geomagnetic Cutoff Rigidities for Epoch 2015
}

\section{F. Smart ${ }^{1}$}

SSSRC

100 Tennyson Avenue, Nashua, NH 03062, USA

donsmart100@msn.com

\section{A. Shea}

SSSRC

100 Tennyson Avenue, Nashua, NH 03062, USA

sssrc@msn.com

\begin{abstract}
Earth's geomagnetic field is evolving rapidly (in geological time), and as a consequence the amount of geomagnetic shielding at a specific location is also changing. Geomagnetic cutoff rigidities derived from the International Geomagnetic Reference Fields (IGRF) are a basic quantity necessary to compute the cosmic radiation exposure at locations around the world. The most recent generation of the IGRF describes Earth's magnetic field to previously unattainable precision. The use of the Epoch 2015 geomagnetic field coefficients in the trajectory-tracing technique gives a more precise set of vertical geomagnetic cutoff rigidity values than previous internal field models. For example, an increase in cutoff rigidity values in the Western North Atlantic Ocean area and eastern North America is the result of the geomagnetic "westward drift".
\end{abstract}

On a world-wide basis the cutoff rigidity values have decreased consistent with a decrease in the magnitude of the geomagnetic dipole term. While requiring an extremely stable neutron monitor over a long time period, neutron monitors in areas of the world with rapidly decreasing cutoff rigidity values should observe an increase in galactic cosmic radiation such as was observed at Huancayo, Peru. Examples of the change in vertical cutoff rigidity values for specific locations including cosmic ray stations are illustrated.

36th International Cosmic Ray Conference -ICRC2019-

July 24th - August 1st, 2019

Madison, WI, U.S.A.

\section{${ }^{1}$ Don F. Smart}


1. Introduction

In geological terms there is a decrease in the main dipole magnetic field by about $5 \%$ per century and there is a westward drift of the non-dipole magnetic field [1]. As the varying regions of total magnetic intensity move, there are changes in the geomagnetic field that modify the cosmic ray access to any specific location on Earth.

We have derived an updated world grid of vertical geomagnetic cosmic ray cutoff rigidities employing the 12th Generation International Geomagnetic Reference Field [2] for Epoch 2015. Vertical geomagnetic cutoff rigidities were calculated each $5^{\circ}$ in latitude and longitude.

\section{Method}

Details of the cosmic ray trajectory calculation procedure have been previously published $[3,4]$ and need not be inclded in this manuscript. In summary, the trajectories of cosmic rays were initiated in the "vertical" direction at an altitude of $20 \mathrm{~km}$ above the surface of the international reference ellipsoid. (The "sensible" atmosphere of the earth was considered to be $20 \mathrm{~km}$.) The cosmic ray orbits were traced (employing a step size that was one percent of a gyro radii distance) until the particle exceeded a radial distance of 25 earth radii, an allowed trajectory, or re-entered the earth, a forbidden trajectory. In the few cases when a trajectory had not reach a solution by 200,000 Runge Kutta steps, a failed trajectory, the particle was considered to be forbidden.

Cutoff rigidities are determined by calculating cosmic ray trajectories through a model of the Earth's magnetic field at discrete rigidity intervals starting with a rigidity value high above the highest possible cutoff and decreasing the rigidity until the lowest allowed trajectory was found. As these calculations progress down through the rigidity spectrum, the results change from the easily allowed orbits to a complex structure of allowed, forbidden, and quasi-trapped orbits (the cosmic penumbra), and finally to a rigidity value below which all trajectories fail to escape the Earth's magnetic field and intersect the solid earth. Rigidity intervals of $0.01 \mathrm{GV}$ were used to provide a reasonable sample of the cosmic ray penumbra. An "effective cutoff rigidity" obtained by summing over the allowed orbits in the penumbra. See [5] for a detailed definition of cosmic ray cutoff rigidities and [6] for a definition of cosmic ray cutoffs.

\section{Results and Discussion}

These calculated geomagnetic cutoff rigidity values are intended to be a reference for evaluating the changes in cutoff rigidity attributable to the evolution of the quiescent internal geomagnetic field. A world map showing the iso-rigidity contours of the effective vertical cutoff rigidities calculated utilizing the International Geomagnetic Reference Field model for Epoch 2015 [2] are presented in Fig. 1 and tabulated $5^{\circ}$ in latitude by $15^{\circ}$ in longitude in Table $1 \mathrm{~A}$ and Table $1 \mathrm{~B}$.

The differences in magnitude of the change in vertical geomagnetic cutoff rigidities (in GV) between Epoch 2000 [7] and Epoch 2015 is shown in Fig. 2. Regions where the cutoff rigidities are increasing are indicated by the black contours; areas where the cutoff rigidities are decreasing are indicated by the red contours. 


\section{VERTICAL CUTOFF RIGIDITIES (GV) 2015 IGRF}

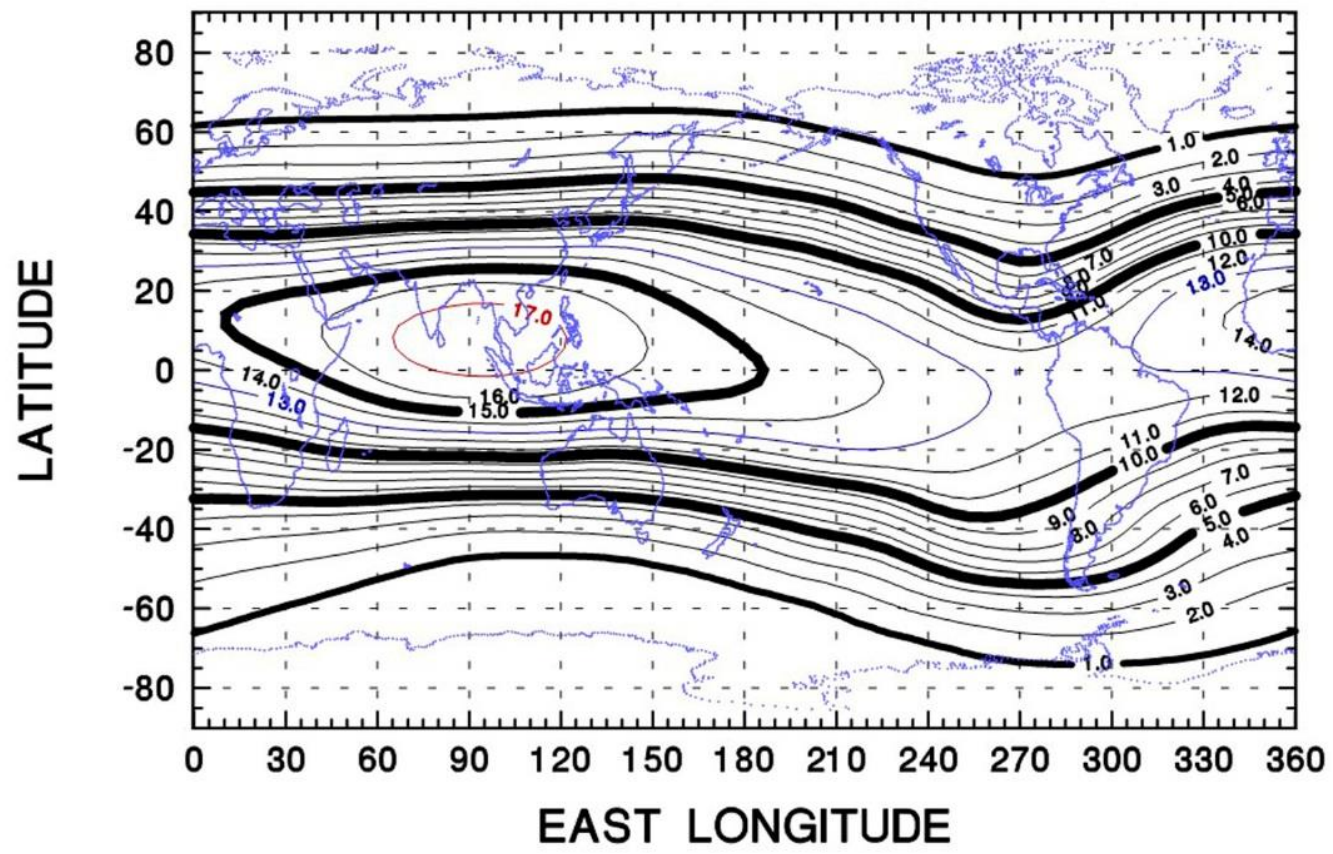

Fig. 1. A world map of vertical cutoff rigidities for epoch 2015.

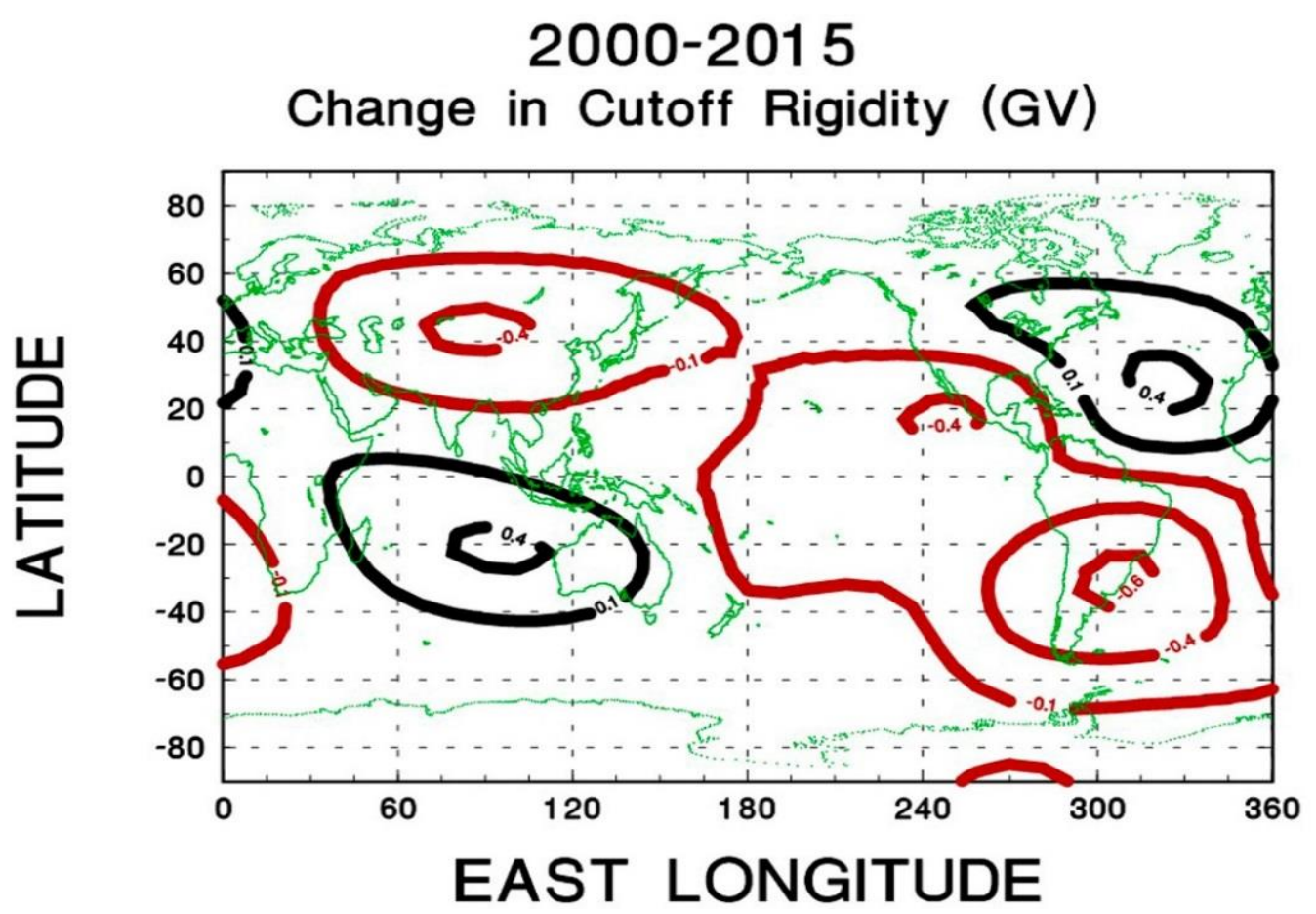

Fig. 2. A map of the change in cutoff rigidities (in GV) between 2000 and 2015.

An examination of Fig. 2 shows that these changes are not uniform over the world, indicating that the non-dipole terms are important for an accurate determination of cutoff rigidities near the surface of the earth. The cutoff rigidities are increasing (indicated 
by the black contours) in the North Atlantic Ocean area. This is the result of the westward movement of non-dipole magnetic field components. The largest increases over this fifteen-year interval are of the order of $0.4 \mathrm{GV}$. Note that there is also an area of increased cutoff rigidity in the Indian Ocean.

The vertical cutoff rigidities are decreasing (indicated by the red contours) in the South Atlantic Ocean area as well as locations in South and Central America extending into the Pacific Ocean. The largest decrease over this fifteen-year interval is of the order of $0.6 \mathrm{GV}$ in Uruguay, South America. This is the combined result of the reduction of the main dipole component of the magnetic field as well as the additional displacement of the effective magnetic center from Earth's geo-center. Researchers in South America [8] are studying the effects of these changes by establishing specific cosmic ray and geomagnetic observatories. Also note that there is a region of decreasing cutoff rigidity in central Asia with a "mirror" region of increases in the same longitude region in the Indian Ocean.

When the changes in cutoff rigidity are expressed in percent (see Fig. 3), we note the continuing changes in Mexico and South America. However, there are also interesting changes in the North Polar Region. Modelers have noted rapid changes (in geological terms) in the location of the magnetic north dip pole and dipole position. The magnetic field modelers have also noted sudden changes in the magnitude of some of the non-dipole components of the magnetic field and these are reported in the literature as geomagnetic jerks $[9,10]$.

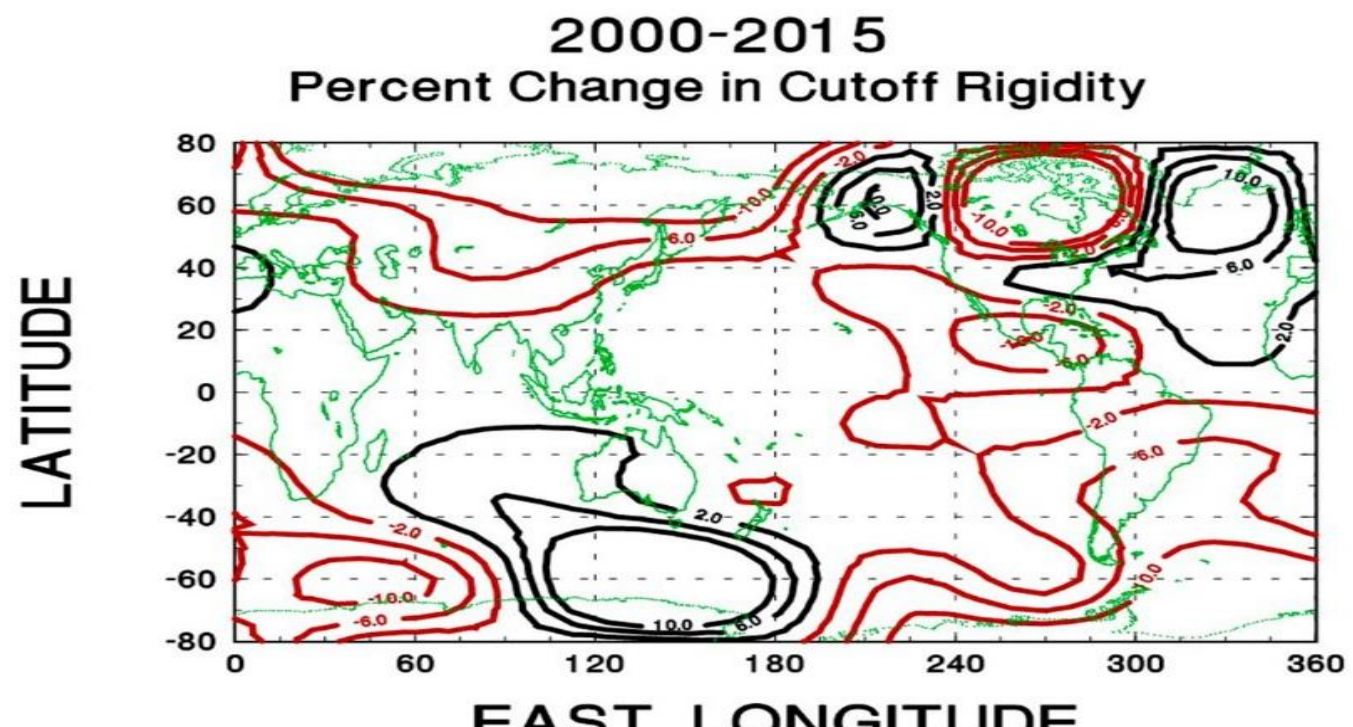

Fig. 3. A map of the change in cutoff rigidities (in percent) between 2000 and 2015. 

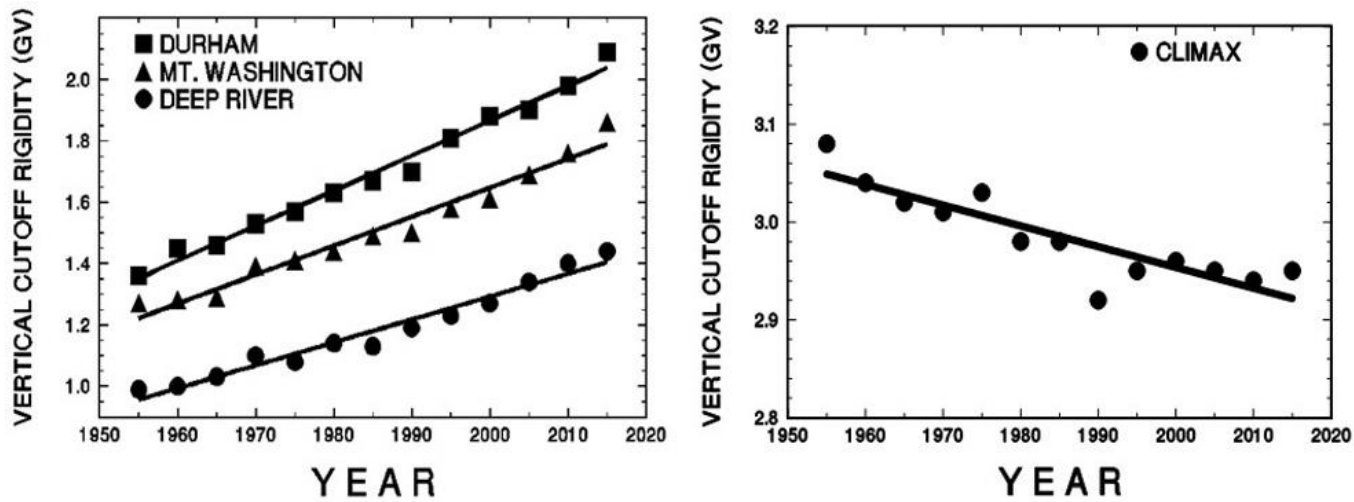

Fig. 4. Change in cutoff rigidity for selected North American Locations.

Table 1 A. 2015 World Grid of Geomagnetic Cutoff Righties.

\begin{tabular}{|c|c|c|c|c|c|c|c|c|c|c|c|c|}
\hline & & & & & \multicolumn{2}{|c|}{ Geographic } & \multirow{2}{*}{$\frac{\text { East }}{90}$} & \multicolumn{2}{|c|}{ Longitude } & \multicolumn{3}{|c|}{ wg2015 } \\
\hline & 0 & 15 & 30 & 45 & 60 & 75 & & 105 & 120 & 135 & 150 & 165 \\
\hline \multicolumn{13}{|l|}{ Lat } \\
\hline 90 & 0.00 & 0.00 & 0.00 & 0.00 & 0.00 & 0.00 & 0.00 & 0.00 & 0.00 & 0.00 & 0.00 & 0.00 \\
\hline 85 & & & & & 0.00 & & & & 0.00 & & 0.00 & 0.00 \\
\hline 80 & & & 0. & & 0.02 & 0.03 & 0.03 & 0.04 & .03 & & 0.04 & .03 \\
\hline 75 & & & & & 0.13 & 0.08 & & & 0.13 & & 0.17 & .13 \\
\hline 70 & & & 0.3 & & 0.35 & 0.41 & 0.38 & & 0.47 & & 0.46 & .43 \\
\hline 65 & & & 0. & & 0.76 & & & & 0.91 & & 1.01 & 0.96 \\
\hline 60 & & & & & 1.3 & 1.44 & & & 1.72 & & 1.86 & 1.80 \\
\hline 55 & & & & & 2.30 & 2.38 & & & 2.75 & & 3.10 & 2.97 \\
\hline 50 & & & & & 3.58 & 3.69 & 3.81 & & 4.30 & & 4.72 & 4.54 \\
\hline 45 & 4. & 5. & & & 5.25 & 5.39 & 5.53 & 5. & 6.03 & & 6.35 & 5.96 \\
\hline 40 & & & & & 7.32 & 7.67 & 7.99 & 8. & 8.93 & & 9.22 & 8.63 \\
\hline 35 & & 9.73 & & & 10.51 & 10.87 & 11.13 & 11.02 & 11.29 & & 11.16 & 10.30 \\
\hline 30 & .73 & 11.77 & 11.79 & 13 & 12.61 & 3.10 & 13.58 & 13.84 & .86 & 13. & 13.19 & 12.51 \\
\hline 25 & & & & & 14.62 & .05 & $15 \cdot 32$ & 35 & .19 & & 4.22 & 13.52 \\
\hline 20 & & & & & 15.76 & 16.23 & 16. & 16. & .15 & & 14.99 & 14.29 \\
\hline 15 & & & & & 16.49 & & 17.25 & 17.15 & .78 & & 15.54 & 14.88 \\
\hline 10 & & & & & 16.82 & 7.36 & 17. & 17. & 08 & & 5.87 & 5.25 \\
\hline 5 & & & & & 16.7 & 7.33 & 17. & 17. & 07 & & 5.97 & 5.49 \\
\hline 0 & & & & & 16.29 & & 17. & 17. & 73 & & 5.81 & 15.46 \\
\hline-5 & & & & & 15.48 & .08 & 16.38 & 16. & .06 & & 5.35 & .15 \\
\hline-10 & & & & & 14.33 & 14.89 & 15.18 & 15.23 & 15.02 & & 4.54 & 14.50 \\
\hline-15 & & 10.36 & 19 & & 12.80 & 13.32 & 13.56 & 13.67 & 13.54 & & 13.29 & 13.42 \\
\hline-20 & & & & & 10.60 & 10.89 & 11. & 11.36 & .19 & & 10.67 & 11.73 \\
\hline-25 & & & & & 8.34 & 8.09 & 7.89 & 7.96 & .02 & & 3.55 & 9.35 \\
\hline-30 & & & & & 5.73 & 5.45 & 5.41 & 5. & 5.45 & & 5.93 & 6.53 \\
\hline-35 & & & & & 4.17 & 3.97 & 3.60 & 3.52 & 3.56 & & 4.22 & 4.84 \\
\hline-40 & & & & 3.38 & 2.90 & 2.57 & 2.26 & 2.12 & 2.19 & & 2.69 & 3.29 \\
\hline-45 & & & & & 1.96 & 1.60 & 1.28 & 1.22 & 1.17 & & 1.55 & 2.03 \\
\hline-50 & 2.32 & 2.07 & 1.84 & 1.59 & 1.24 & 0.94 & 0.71 & 0.58 & 0.57 & & 0.85 & 1.18 \\
\hline-55 & & & & 1.07 & 0.81 & 0.54 & 0.34 & 0.20 & 0.22 & & 0.36 & 0.61 \\
\hline & & & & & 0.49 & 0.28 & 0.11 & & 0.03 & & 0.12 & 0.25 \\
\hline-65 & & & & & 0.20 & 0.12 & 0.03 & & 0.00 & & 0.02 & 0.06 \\
\hline-70 & & 0.55 & 0.39 & 0.24 & 0.09 & 0.03 & 0.00 & 0.00 & 0.00 & 0.00 & 0.00 & 0.00 \\
\hline & & & & & 0.04 & 0.00 & 0.00 & 0. & 0.00 & & 0.00 & 0.00 \\
\hline-80 & & & & 0.06 & 0.03 & 0.00 & 0.00 & & 0.00 & & 0.00 & 0.00 \\
\hline-85 & 0.12 & 0.07 & 0.06 & 0.02 & 0.02 & 0.02 & 0.00 & 0.00 & 0.00 & 0.00 & 0.00 & 0.00 \\
\hline-90 & 0.04 & 0.04 & 0.04 & 0.04 & 0.04 & 0.04 & 0.04 & 0.04 & 0.04 & 0.04 & 0.04 & 0.04 \\
\hline
\end{tabular}


Table 1 B. 2015 World Grid of Geomagnetic Cutoff Righties.

\begin{tabular}{|c|c|c|c|c|c|c|c|c|c|c|c|c|}
\hline \multirow{2}{*}{\multicolumn{2}{|c|}{ Lt/Lon 180}} & \multirow[b]{2}{*}{195} & \multirow[b]{2}{*}{210} & \multirow[b]{2}{*}{225} & \multicolumn{2}{|c|}{ Geographic } & \multirow{2}{*}{$\begin{array}{r}\text { East } \\
270 \\
\end{array}$} & \multicolumn{2}{|c|}{ Longitude } & \multicolumn{3}{|c|}{ wg2015 } \\
\hline & & & & & 240 & 255 & & 285 & 300 & 315 & 330 & 345 \\
\hline \\
\hline 90 & 0.00 & 0.00 & 0.00 & 0.00 & 0.00 & 0.00 & 0.00 & 0.00 & 0.00 & 0.00 & 0.00 & 0.00 \\
\hline 85 & & & & & 0.00 & & & 0.00 & 0.00 & & 0.00 & 0.00 \\
\hline 80 & & & 0.0 & & 0.00 & 0.00 & 0.00 & 0.00 & .00 & & .00 & .00 \\
\hline 75 & & & & & & & 0.00 & 0.00 & .00 & & .02 & .05 \\
\hline 70 & & & & & 0.00 & 0.00 & 0.00 & 0.00 & .02 & & .15 & .20 \\
\hline 65 & & & & & 0.07 & 0.04 & 0.05 & 0.06 & .15 & & .45 & .58 \\
\hline 60 & & & & & 0.32 & 0.21 & 0.22 & 0.31 & .49 & & .94 & .14 \\
\hline 55 & & & & & 0.65 & 0.50 & 0.50 & 0.65 & .94 & & 1.75 & 2.02 \\
\hline 50 & & & & & 1.1 & 0.5 & 0 . & 1.21 & .71 & & .96 & 3.24 \\
\hline 45 & & & & & 1.9 & 1.57 & & 1.94 & .85 & & 4.60 & 4.97 \\
\hline 40 & 72 & 5. & $4 . \varepsilon$ & & 2.99 & 2.46 & 2.43 & 3.00 & .24 & & 6.56 & 7.24 \\
\hline 35 & & & & & & & & & .87 & & .54 & 9.86 \\
\hline 30 & & & & & 5. & & & 5.76 & .89 & 10. & 1.40 & 11.71 \\
\hline 25 & 12.32 & 61 & 10.73 & 53 & 7.80 & 6.22 & 6.14 & 8.09 & 10.88 & 12.19 & 12.93 & 13.35 \\
\hline 20 & & & 1.9 & & 8.5 & 6.99 & 4 & 9.87 & .07 & & 3.73 & 14.18 \\
\hline 15 & & & 2.79 & & 10.64 & 9.38 & 9. & 11.59 & .74 & & 14.10 & 14.57 \\
\hline 10 & & & 3.44 & .83 & 11.92 & 11.10 & 11.33 & 12.25 & .07 & 66 & 14.10 & 14.54 \\
\hline 5 & & & & & 12.76 & 2.16 & 12.12 & 12.57 & .13 & & .75 & 14.14 \\
\hline 0 & & & 2 & & 13.17 & 12.66 & 12.43 & 12.65 & .95 & & 3.11 & 13.41 \\
\hline-5 & & & 4.14 & 75 & 13.31 & 12.83 & 12.52 & 12.53 & 12.57 & .40 & 12.26 & 12.43 \\
\hline-10 & & & 5 & & 13.25 & 12.79 & 12.41 & 12.24 & 12.02 & 11. & 11.15 & 11.23 \\
\hline-15 & & & 3.54 & & 13.00 & 12.58 & 12.14 & 11.79 & .34 & & 9.87 & .80 \\
\hline-20 & .60 & 12.84 & 2.91 & 2.83 & 12.60 & 12.21 & 11.73 & 11.21 & 10.45 & & .49 & .13 \\
\hline-25 & 10.25 & 11.34 & 20 & 12.14 & 12.04 & 11.72 & 11.19 & 10.40 & 9.50 & & 7.18 & .86 \\
\hline-30 & & & 10.03 & 11.22 & 11.31 & 11.10 & 10.47 & 9. & 42 & & 6.22 & 5.61 \\
\hline-35 & 02 & .21 & & 9.01 & 10.42 & 10.27 & 9.66 & 8.70 & .30 & & 5.20 & 1.49 \\
\hline-40 & 4.53 & .17 & & 7.75 & 9.07 & 9.23 & 8.68 & 7.62 & .57 & & 4.34 & 3.68 \\
\hline-45 & & & & & 7.23 & 8.03 & 7.82 & 7.02 & 5.80 & & 3.56 & 3.11 \\
\hline-50 & .02 & 2.66 & & & 5.04 & 5.92 & 6.28 & 5.52 & 4.50 & & 2.93 & 2.49 \\
\hline-55 & 1.18 & 1.68 & 2.3 & 3.04 & 3.87 & 4.37 & 4.51 & 4.24 & 3.64 & & 2.31 & 1.92 \\
\hline-60 & 0.67 & 1.04 & 1.53 & 2.02 & 2.62 & 3.15 & 3.46 & 3.24 & 2.78 & 2. & 1.94 & 1.47 \\
\hline & & & & & 1.73 & 2.06 & 2.34 & 2.31 & 2.02 & & 1.39 & 1.14 \\
\hline-70 & & & & & 1.03 & & 1.42 & 1.46 & 1.37 & & 1.00 & 0.79 \\
\hline-75 & 0.03 & 0.17 & 0.24 & 0.43 & 0.60 & 0.72 & 0.80 & 0.84 & 0.85 & 0.72 & 0.65 & 0.51 \\
\hline & & & & 0.2 & 0.32 & 0.38 & 0.44 & 0.43 & 0.44 & 0. & 0.37 & 0.29 \\
\hline-85 & & & & & 0.07 & 0.11 & 0.11 & 0.18 & 0.16 & & 0.16 & 0.16 \\
\hline-90 & 0.04 & 0.04 & 0.04 & 0.04 & 0.04 & 0.04 & 0.04 & 0.04 & 0.04 & 0.04 & 0.04 & 0.04 \\
\hline
\end{tabular}
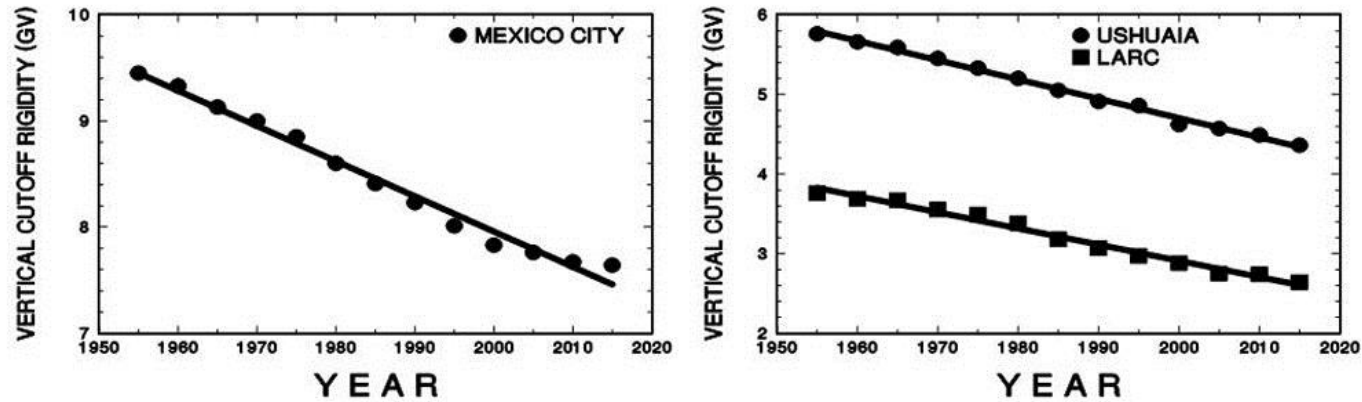

Fig. 5. Change in cutoff rigidity for Mexico and selected South American Locations. 

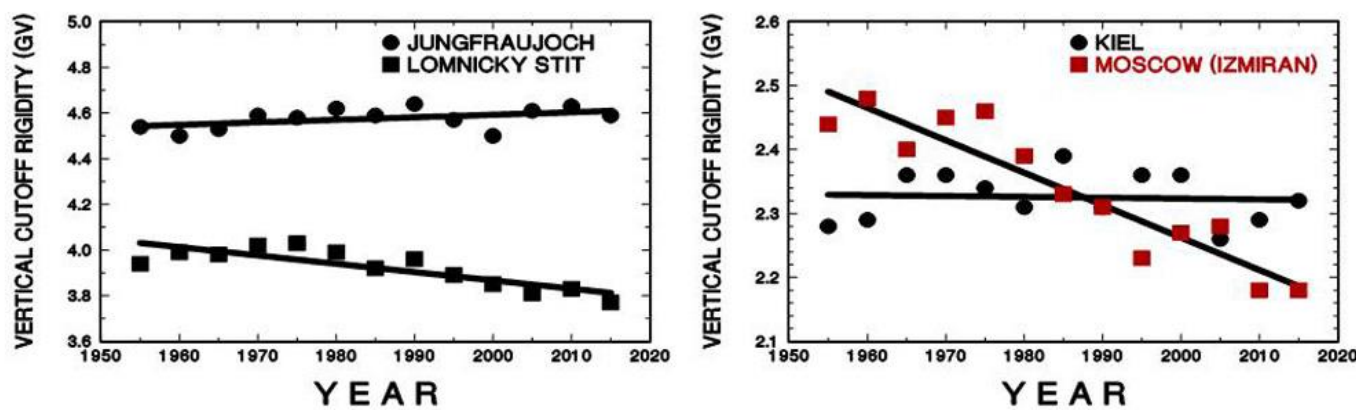

Fig. 6. Change in cutoff rigidity for selected European Locations.
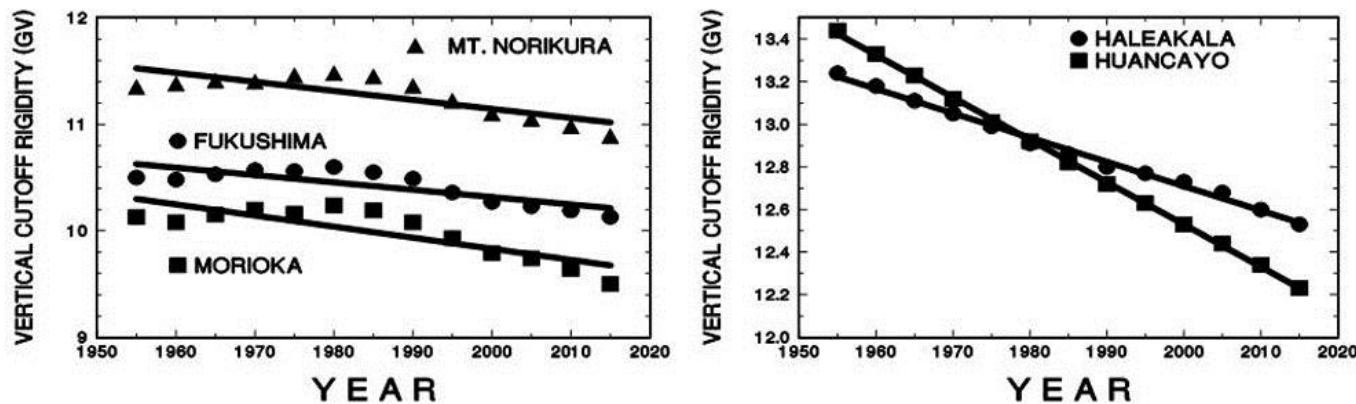

Fig. 7. Change in cutoff rigidity for selected high rigidity locatiions.

\section{Summary}

The new world grid of vertical cutoff rigidities for Epoch 2015 shows significant and systematic changes from the previous epochs. A careful inspection of a world grid of vertical geomagnetic cutoff rigidities shows that the westward drift [1] of the non-dipole components of Earth's magnetic field results in an increase in cutoff righties along the North American east coast region while there is a continuing decrease in the South American region. The evolution of these non-dipole components can have a "local effect" on the cutoff rigidity of some cosmic ray stations.

\section{References}

[1] R. Merrill, M. W. McElhinny and P McFadden, The Magnetic Field of the Earth: Paleomagnetism, the Core and the Deep Mantel, 63, International Geophysics Series (1996) Academic Press.

[2] E. Thébault et al., International Geomagnetic Reference Field: the 12th generation, Earth, Planets and Space, 67 (2015) 67-79 doi:10.1186/s40623-015-0228-9

[3] D. F. Smart, M.A. Shea and E.O. Flückiger, Magnetospheric Models and Trajectory Computations, Space Sci. Rev., 93, (2000) 305-333.

[4] D. F. Smart and M, A. Shea, Geomagnetic Cutoff Rigidity Computer Program, Final Report, Grant NAG5-8009, Center for Space Plasmas and Aeronomic Research, University of Alabama in Huntsville (2000). 
[5] M. A. Shea, D. F. Smart and K. G. McCracken, A Study of Vertical Cutoff Rigidities Using Sixth Degree Simulations of the Geomagnetic Field, J. Geophys. Res., 70 (1965) 41174130.

[6] D. J. Cooke, et al., On Cosmic-Ray Cut-Off Terminology, II Nuovo Cimento C, 14, (1991) 213-234.

[7] D. F. Smart and M. A. Shea, World Grid of Calculated Cosmic Ray Vertical Cutoff Rigidities for Epoch 2000.0, 30th International Cosmic Ray Conference, (2007). (On CD-Rom, available www.icrc2007.unam.mx.)

[8] E. G. Cordaro, P. Venegas-Aravena, and D. Laroze, Variations of geomagnetic cutoff rigidity in the southern hemisphere close to $70^{\circ} \mathrm{W}$ (South-Atlantic Anomaly and Antarctic zones) in the period 1975-2010, Advances in Space Research, 63 (2019) 2290-2299.

[9] K. J. Pinneiro, A. Jackson and C. C. Finlay, Measurements and uncertainties of the occurrence time of the 1969, 1978, 1991 and 1999 geomagnetic jerks, Geochem. Geophys. Goesyst, 12 (2011). Q10015

[10] W. Brown, J. Mound, and P. Livermore, Jerks abound: an analysis of geomagnetic observatory data from 1957 to 2008, Phys. Earth Planet. Int., 223, (2013) 62-76 